\title{
Prevalencia coprológica de parásitos gastrointestinales en humanos y porcinos de crianza de traspatio del distrito de Zapatero, San Martín
}

\author{
Coprological prevalence of gastrointestinal parasites in \\ human and Backyard breeding pigs population in Zapa- \\ tero District, San Martín \\ Puicón, Victor ${ }^{10000-0003-2532-2551]}$; López-Flores, Alicia ${ }^{1[0000-0002-4679-6353]}$; \\ Fabian-Dominguez, Fredy ${ }^{1[0000-0003-3577-5896]}$ y Sánchez-Cárdenas, \\ Hugo ${ }^{1[0000-0003-1560-2402]}$ \\ ${ }^{1}$ Laboratorio de Histopatología, Escuela de Medicina Veterinaria \\ ${ }^{1}$ Universidad Nacional de San Martín, Tarapoto, Perú \\ vhpuicon@unsm.edu.pe
}

Resumen. El objetivo del presente estudio de investigación fue determinar la prevalencia de parasitismo intestinal en porcinos y humanos del Centro Poblado Menor de Bagazan, provincia de Lamas, Perú. Se colectaron 176 muestras fecales de porcinos de crianza de traspatio y 179 muestras de población humana relacionada a la crianza de estos animales. Las muestras fueron colectadas independientemente de la edad y sexo y fueron analizadas cualitativamente mediante la técnica parasitológica de flotación con solución sobresaturada de cloruro de sodio y la estimación de la carga parasitaria se realizó con el método de McMaster modificado. La prevalencia de parasitismo intestinal en los cerdos fue $28.41 \%$ (50/176), encontrándose Eimeria spp (63.07\%), huevos tipo Strongylus (26.71\%), Ascaris suum (12.5\%), Macracanthorhynchus hirudinaceus (1.14\%), Trichuris suis $(0.57 \%)$ y Balantidium coli $(0.57 \%)$. En el caso de los humanos, la prevalencia fue $91.6 \%$, encontrándose Ascaris lumbricoides (34.09\%), Strongyloides stercoralis $(1.11 \%)$, Trichuris trichiura $(2.72 \%)$ y Enterobius vermicularis $(0.55 \%)$. No se determinó asociación significativa entre prevalencia parasitaria y los factores sexo y edad en porcinos.

Citar como: Puicón, V., López-Flores, A., FabianDominguez, F. \& SánchezCárdenas, H. (2021).

Prevalencia coprológica de parásitos gastrointestinales en humanos y porcinos de crianza de traspatio del distrito de Zapatero, San Martín. Revista de Veterinaria y Zootecnia Amazónica, 1(1), 4-14. https://doi.org/10.51252/re vza.v1i1.127

Recibido: 02/04/2021

Aceptado: 05/06/2021

Publicado: 28/07/2021
Palabras clave: Bagazan, cerdos, coproparasitología, parásitos

Abstract. The aim of this study was to determine the prevalence of intestinal parasitism in pigs and humans population from the Centro Poblado Menor de Bagazan, Lamas province, Peru. In total, 176 faecal samples were collected from backyard pigs and 179 samples from people related to the raising of these animals. The samples were collected regardless of age and sex and were qualitatively analysed using the parasitological technique of flotation with supersaturated sodium chloride solution and the estimation of the parasite load was made with the modified McMaster method. The prevalence of intestinal parasitism in pigs was $28.41 \%$ (50/176), with Eimeria spp (63.07\%), Strongylus-type eggs (26.71\%), Ascaris suum (12.5\%), Macracanthorhynchus hirudinaceus (1.14\%), Trichuris suis $(0.57 \%)$ and Balantidium coli $(0.57 \%)$. In the case of humans, the prevalence was $91.6 \%$, with Ascaris lumbricoides $(34.09 \%)$, Strongyloides stercoralis $(1.11 \%)$, Trichuris trichiura $(2.72 \%)$ and Enterobius vermicularis $(0.55 \%)$. No significant association between parasite prevalence and sex and age factors in pigs was determined.

Keywords: Bagazan, coproparasitology, parasites, pigs 


\section{$1 \quad$ Introducción}

En los últimos años, la importancia de las enfermedades parasitarias, comunes a porcinos y humanos, ha ido incrementándose, en nuestro país, el $10.6 \%$ de la producción porcina se encuentra ubicada en la zona selva, siendo el 79.2\% de tipo criollo (1). En términos generales, los sistemas de producción porcina varían desde la crianza tecnificada hasta la crianza de traspatio como forma secundaria a otras actividades familiares. La incidencia de parasitismo es mayor debido a causas como malas condiciones de sistema de gestión, vivienda, medidas higiénico-sanitarias, ubicación geográfica y edad de los animales (2).

El principal problema de las parasitosis en porcinos es su potencial de generar infecciones gastrointestinales e infestaciones tisulares, muchas de las cuales representan riesgo para la salud humana (3). Los cerdos se ven afectados por una reducción en el consumo de alimentos, bajos índices de conversión alimenticia, anemia, diarrea y pérdida de proteínas plasmáticas a nivel del tracto gastrointestinal (4), además de un aumento en la susceptibilidad a la presentación de enfermedades de origen bacteriano o viral, e incremento en costos por el uso de antihelmínticos, complejos vitamínicos, etc. (5). Por otro lado, las consecuencias perceptibles en los productores se evidencian con pérdidas económicas debido a una disminución de los parámetros productivos tales como menor ganancia de peso, mayor tiempo de engorde o por el decomiso de órganos, como el caso del hígado, debido a migraciones larvarias de helmintos (5).

Los habitantes de la comunidad del Centro Poblado Menor de Bagazán se dedican a la agricultura, siendo su principal fuente de ingreso la producción de plátanos y la cría de cerdos como medio de subsistencia. En esta comunidad no se han realizado estudios sobre parasitismo gastrointestinal ni en porcinos ni en humanos, sin embargo, se reporta que comunidades con bajos ingresos económicos enfrentan mayores riesgos de presentar infecciones parasitarias tisulares como cisticercosis (6).

En la región Selva y específicamente en la región San Martín, los estudios realizados en porcinos, se limitan y se enfocan en temas de producción, siendo escasos los estudios realizados en parasitología en porcinos. Sin embargo, un estudio realizado por (7) indican que las provincias Huallaga, Mariscal Cáceres, Lamas y San Martín de la Región de San Martín, se reportaron helmintos que actualmente engloban a un solo conjunto de parásitos denominados HTS, los cuales conforman las especies Trichostrongylus axei, Oesophagostomun dentatum, Stephanurus dentatus, Hiostrongylus rubidus y Globocephalus urosubulatus. En otras regiones de nuestro país como en la sierra central, Jauja, Junín, se reporta un $22.57 \%$ de prevalencia parasitaria (8), en relación a protozoarios, la prevalencia de Balantidiun coli en la población porcina del distrito de Acora fue elevada (88,75\%) (9). 
En Cundimarca, Colombia, se realizó un estudio tanto en granjas porcinas tecnificadas y semitecnificadas como en humanos, obteniéndose como resultados, la presencia de protozoarios como Cryptosporidium spp, Balantidium coli, Endolimax nana, Giardia spp., Idomaeba bütschlii, en tanto que nematodos como Ascaris suum, Trichuris suis y Strongyloides spp. En el mismo estudio, en humanos se hallaron así mismo, protozoarios como E. coli, E. hystolitica/dispar, B. coli, B. hominis e I. bütschlii (10).

En estudios realizados en otras localidades del mundo sobrepasan el porcentaje de prevalencia de 65\%, tales como en países sudamericanos (Estado de Falcón, Venezuela) (11), europeos (Dinamarca) (12), asiáticos (Chungcheongnam-do, Corea de Sur) (13), (Mumbai, India) (14) y africanos como Kabale, Uganda (15).

El presente estudio tuvo como objetivo determinar las especies y/o géneros de los parásitos gastrointestinales y su respectiva prevalencia en porcinos y humanos del distrito de Zapatero. Así mismo, se determinó la influencia entre las variables edad y sexo de los porcinos en la frecuencia de parásitos gastrointestinales.

\section{Materiales y Métodos}

El presente estudio se realizó entre marzo y junio de 2019 en el centro poblado de Bagazán, distrito de Zapatero, provincia de Lamas, Región San Martín, Perú. La zona presenta una temperatura promedio de $22{ }^{\circ} \mathrm{C}$ y una precipitación pluvial anual de $670 \mathrm{~mm}$. El tamaño de muestra fue obtenido a conveniencia; es decir, se trabajó con la totalidad de los porcinos criados en traspatio y con los habitantes que crían o consumen cerdos.

En relación de que la población rural de porcinos fue baja, se consideró la totalidad de 176 cerdos criollos, desde 6 a 18 meses de edad y de ambos sexos. Además, 179 habitantes adultos de ambos géneros, no desparasitados en un tiempo menor a 1 mes. La toma de muestra en porcinos se realizó extrayendo digitalmente a nivel rectal a primeras horas de la mañana, posteriormente las muestres fueron almacenadas en cajas de tecnopor con geles refrigerantes hasta su transporte al Laboratorio de Sanidad Animal de la Escuela de Medicina Veterinaria de la Universidad Nacional de San Martín. Las muestras en humanos fueron colectadas por los mismos habitantes, a los cuales se les brindó un frasco colector, para luego ser trasladadas para su procesamiento.

Las muestras fueron analizadas mediante las técnicas cualitativas de sedimentación (16) y la técnica cuantitativa de McMaster modificado. Los huevos de los nematodos Trichostrongylus axei, Oesophagostomum dentatum, Stephanurus dentatus, Hyostrongylus rubidus y Globocephalus urosubulatus, fueron denominado en su conjunto como Huevos tipo Strongylus (HTS) dada su común morfología. 
Se determinó el número promedio de parásitos por porcino y humano (carga parasitaria) y la prevalencia de la parasitosis, expresándose en forma porcentual. Se consideró como muestra positiva aquella muestra fecal que contenga al menos un agente parasitario (17). Para hallar relaciones significativas de esta parasitosis en porcinos frente a las variables sexo y edad, se realizó una estratificación por edades y luego se aplicó un análisis de Chi cuadrado mediante el paquete estadístico SPSS Statistics v. 25.0.

\section{Resultados y discusiones}

En nuestro país y en la región San Martin, los estudios en relación a las parasitosis gastrointestinales en cerdos son limitados, puesto que los enfoques en esta especie conciernen mayormente a la teniasis-cisticercosis, cuya fase de neurocisticercosis representa un grave problema en salud pública.

La prevalencia global de parásitos en cerdos fue 28.4\% (50/176). Las frecuencias según el agente parasitario se presentan en el Tabla 1, donde el agente con mayor frecuencia correspondió a Eimeria spp (63.07\%). El promedio de huevos por gramo de heces (hpg) en HTS fue de 77.84, teniéndose individuos con cargas máximas de hasta $2450 \mathrm{hpg}$. El promedio de huevos de Ascaris suum fue 247.7, y con individuos con carga máxima de $6200 \mathrm{hpg}$. El grado de infección parasitaria de Eimeria spp fue predominantemente leve (+) (41/111). No se hallaron formas parasitarias correspondientes a trematodos ni en cerdos ni en humanos.

Tabla 1.

Especies parasitarias halladas en cerdos del distrito de Zapatero, San Martín (n=176)

\begin{tabular}{lr}
\hline \multicolumn{1}{c}{ Especie } & Positivos (\%) \\
\hline Eimeria spp & $63.07 \%$ \\
HTS $^{1}$ & $26.71 \%$ \\
Ascaris suum & $12.50 \%$ \\
Macracanthorhynchus hirudinaceus & $1.14 \%$ \\
Trichuris suis & $0.57 \%$ \\
\hline
\end{tabular}

En el presente estudio se obtuvo, una prevalencia global de parásitos en porcinos del centro poblado de Bagazan de $28.41 \%$ (50/176), cifra menor que las reportadas para otras ciudades dentro de nuestro país como Jauja, Junín (22.57\%) (8) y de otras localidades del mundo que sobrepasan el 65\%, tales como en países sudamericanos (Estado de Falcón, Venezuela) (11), europeos (Dinamarca) (12), asiáticos (Chungcheongnam-do, Corea de Sur) (13), (Mumbai, India) (14) y africanos como Kabale, Uganda (15).

En relación a las prevalencias especificas por el tipo de parasito, se reportó en el presente estudio una prevalencia coprológica de $12.50 \%$ para Ascaris suum, un reporte de cifra más baja que lo hallado en Jauja, Junín (22.57\%) (8), Mocupe, Lambayeque (28.21 \%) (18), y muy en 
contraste con las tasas de infección de mayor del 65\% en las halladas por (20), en el cual realizó un estudio postmortem, hallando un $68.53 \%$ de animales infestados por este parasito en un matadero municipal en Huánuco, en tanto que cifra similar de 65,26\%, se halló en el distrito de Laredo, La Libertad (19).

Cabe indicar que aunque el análisis estadístico revela que no existe significancia entre edad y parasitismo, la mayor parte de la muestra analizada eran animales mayores de 6 meses, lo cual podría explicar porque se obtuvo una moderada a baja prevalencia global, tratándose de animales de traspatio, puesto que la mayoría de infecciones ascaroideas afectan a animales menores de 5 meses de edad y con una alimentación carentes de nutrientes (5), puesto que en el distrito, se cuantificó que el 58,8\% de porcinos se alimentan con desechos domésticos, además de registrarse índices socioeconómicos malos en el distrito (20).

Se determinó además una carga parasitaria promedio de 247.72 hpg, sin embargo, los animales que tuvieron mayores cargas de hasta 6200 hpgh tenían 1 año de edad, siendo casos de cerdos con desnutrición y baja condición corporal, los cuales son los más susceptibles a la infección parasitaria considerándose grado severo hasta un recuento de 5000 hpgh (5).

En el caso de humanos, la prevalencia de parásitos fue $91.6 \%$, correspondiendo la mayor frecuencia a la especie de Ascaris lumbricoides (34.09) (Tabla 2). Cuyo promedio de hpg fue de 198.3, teniéndose individuos con cargas máximas de hasta 5800 hpg.

Tabla 2.

Especies parasitarias halladas en humanos del distrito de Zapatero, San Martín (n=179)

\begin{tabular}{lr}
\hline \multicolumn{1}{c}{ Especie } & Positivos (\%) \\
\hline Ascaris lumbricoides & $34.09 \%$ \\
Trichuris trichiura & $2.72 \%$ \\
Strongyloides stercolaris & $1.11 \%$ \\
Enterobius vermicularis & $0.55 \%$ \\
\hline
\end{tabular}

La importancia de la infección cruzada de Ascaris sp. no debe subestimarse, puesto que en el presente estudio se estimó un $34.09 \%$ para Ascaris lumbricoides en la muestra de individuos relacionados a la crianza de traspatio del distrito de Bagazan, obteniéndose personas con cargas máximas de hasta 5800 hpgh. Prevalencias que tanto en humanos y cerdos podrían estar interrelacionadas por la falta de medidas higiénico sanitaria en crianzas de traspatio debido a la mala situación de los factores económicos; puesto que se ha reportado y comprobado la transmisión cruzada de estas especies entre humanos y cerdos (20,21), así mismo, en los últimos estudios, (23) reportaron que poblaciones del género Ascaris sp. obtenidas en humanos y cerdos en diferentes regiones de Brasil no fueron posibles ser discriminables, a través de los marcadores genéticos utilizados, lo que indicó el potencial de transmisión zoonótica y la 
necesidad de un mejor control de estas infecciones en las porquerizas, principalmente en las de traspatio.

Cabe indicar que, entre ambas especies, se halla una interrelación evidentemente evolutiva, la cual ha sido demostrada a través de estudios de resecuenciamiento de todo el genoma, indicando que Ascaris lumbricoides es más primitivo que Ascaris suum (24). Si bien, este estudio carece de un soporte de estudio genético molecular, sugiere que ambas prevalencias podrían tener una relación con el comportamiento tanto antropozoonótico como zooantroponótico del Ascaris sp. Esto se indica en virtud de que existen evidencias genéticas y epidemiológicas de investigación que han demostrado la transmisión zoonótica de las especies de Ascaris sp. en otras regiones del mundo (21),21).

En relación a otros helmintos, los nematodos Huevo tipo Strongylus presentaron una prevalencia de $26.71 \%$, muy distante de lo reportado en Jauja (73.15\%), en el cual, (8) determinó que la carga elevada de HTS es debido a principalmente a que se trabajó con animales de edad mayor de 7 meses y amarrados, a diferencia del presente estudio, en el cual solo el $4 \%$ de los pobladores crían a los cerdos amarrados, mientras que el $24 \%$ los crían sueltos sin control y el $64 \%$ de los criadores de cerdos mencionan que crían los cerdos sueltos pero encerrados en un corral (20).

Con relación a Trichuris suis, en el presente estudio se obtuvo una prevalencia de $0.57 \%$, muy por debajo de los valores reportados en otras ciudades locales del país como Jauja, Junín (18.68 $\%$ ) (8), La Libertad (30.52 \%) (19) y en otras localidades centroamericanas como Managua, Nicaragua (16.6\%) (25) y León, Nicaragua (45.92 \%) (26). Cabe resaltar que en los estudios donde se reporta mayor prevalencia de este parasito, es hallado en mayor carga en animales menores de 6 meses muestreados, puesto que son los más susceptibles hasta inclusive el triple que los animales mayores $(8,5)$, esto podría explicar la baja prevalencia puesto que todos los animales muestreados tenían mayor o igual a 6 meses de edad.

La coccidiosis es un problema muy frecuente a nivel de la producción animal, en el presente estudio se reportó como el parasito de mayor prevalencia a Eimeria spp. (63.07\%) con un grado de infección parasitaria de intensidad leve $(+)$, esta cifra de notable presencia en el presente estudio, concuerda con lo reportado por las otras investigaciones realizadas por (19) $(82,25 \%)$, pero mayor que lo reportado en otros lugares como en Nicaragua (46\%) (25). Estas altas prevalencias se deben a que los animales muestreados, en gran proporción son jóvenes, además la época de estudio fue en época de lluvias, por lo que, en esta región tropical, existe una mayor contaminación de alimentos y mayor índice de humedad de los pastos, factores favorables para la transmisión y desarrollo del parasito (5). 
La prevalencia del ciliado Balantidium coli es ostensiblemente baja en el ganado porcino $(0.57 \%)$ y nula en la población humana, resultados en la que pareciera apoyar los hallazgos de un estudio realizado en el distrito de Acora, Puno publicado por (9), el cual es similar al presente trabajo. Éste indica que la población humana presenta bajas tasa de infección por Balantidium coli, pero difiere en relación a los valores hallados en porcinos, en el cual se reporta una prevalencia alta de $88.75 \%$ en porcinos, este hallazgo, pueda tener como posible explicación a la no detección de este protozoario debido al no haberse procedido en realizar exámenes coprológicos seriados y no haber usado una técnica de laboratorio más específica para protozoarios, puesto que el $\mathrm{NaCl}$ utilizado en la técnica de flotación, genera distorsión y alteración morfológica del parasito.

En cuanto a la acantocefaliasis intestinal, se reportó una prevalencia muy baja de Macracanthorhynchus hirudinaceus (1.14\%), cifra similar a lo hallado en La Libertad, por Arellano (19) (3.68\%), en contraste, con lo reportado de hallazgos a nivel macroscópico en Huánuco (20.53\%) (27), es probable que la baja de estas cifras en lo reportado a nivel coprológico se deba a que los huevos de este parasito son muy pesados (26) por lo que es difícil hallarlo en las técnicas realizadas en el presente estudio, además, cabe resaltar que esta parasitosis tiene un hospedero intermedio de escarabajos, cuya presencia influye en la prevalencia de los mismos (5).

En el presente estudio, no se evidenció una relación estadísticamente significativa $(\mathrm{p}<0.05)$ entre parasitismo y factores como sexo y edad, sin embargo, cabe indicar que en otros estudios como el realizado por Diaz (18) en Mocupe, Lambayeque, reporta que en el caso de Ascaris suum, la edad de los animales oscilo entre 1 - 24 meses, encontrando una asociación de mayor prevalencia en porcinos de $1-9$ meses con 64 casos positivos lo que corresponde a $34.04 \%$ de prevalencia. En el caso de los humanos no fue posible realizar este análisis debido a un desbalance de los datos dentro de cada variable.

En virtud de lo discutido, se recomienda que las autoridades deben continuar con políticas de Salud pública, mejorando las condiciones socioeconómicas de los habitantes del Centro poblado de Bagazan, distrito de Zapatero de tal modo que la población cuente con agua potable, desagües así como la realizaciones de estrategias de apoyo para los agricultores y criadores en el manejo de porcinos de traspatio, favoreciendo la producción y rendimiento de los animales, y disminuyendo las probabilidades de adquirir enfermedades potencialmente zoonóticas.

\section{Conclusiones}

La prevalencia general en porcinos fue $28.41 \%$ (50/176): Eimeria spp. (63.07\%), Huevo tipo Strongylus (26.71\%), Ascaris suum (12.5\%), Macracanthorhynchus hirudinaceus (1.14\%). Trichuris suis $(0.57 \%)$ y Balantidium coli $(0.57 \%)$. 
La prevalencia general en humanos fue 91.6\%: Ascaris lumbricoides (34.09\%), Strongyloides stercolaris (1.11\%), Trichuris trichiura (2.72\%) y Enterobius vermicularis $(0.55 \%)$.

No se determinó asociación significativa entre prevalencia parasitaria y los factores sexo y edad en porcinos $(\mathrm{p}<0.05)$.

\section{Referencias bibliográficas}

1. INEI. Resultados definitivos [Internet]. IV Censo Nacional Agropecuario. 2012.

Disponible en:

http://proyectos.inei.gob.pe/web/DocumentosPublicos/ResultadosFinalesIVCENAGRO. pdf

2. Frontera E, Pérez E, Reina D, Alcaide M. Patología parasitaria porcina: en imágenes. Biomedia ZS-GA, editor. 2009.

3. Valle Peguero Y, Guerra Llorens Y, Mencho Ponce JD, Vázquez Flores A. Comparación del parasitismo gastrointestinal en cerdos estatales y privados en diferentes categorías. Rev Prod Anim [Internet]. 2006;18(2):141-4. Disponible en: https://revistas.reduc.edu.cu/index.php/rpa/article/view/3107

4. Rodríguez-Vivas R, Cob-Galera L, Domínguez-Alpizar J. Frecuencia de parásitos gastrointestinales en animales domésticos diagnosticados en Yucatán, México. Rev Biomédica. 1 de enero de 2001;12(1):19-25. doi:10.32776/revbiomed.v12i1.253

5. Quiroz Romero H. Parasitología y enfermedades parasitarias de animales domésticos. Primera Ed. 2008.

6. Pinto PSA, Santos WLM, Laerte PA, Acevedo-Nieto EC, Santos TO, Duarte CTD. Perfil epidemiológico da cisticercose bovina e suína em três regiões do estado de Minas Gerais, Brasil. Arq Bras Med Veterinária e Zootec. febrero de 2019;71(1):167-76. doi:10.1590/1678-4162-10235

7. Sarmiento L, Tantalean M, Huiza A. Nemátodos parásitos del hombre y de los animales en el Perú. Rev Peru Parasitol [Internet]. 1999;14(2):9-65. Disponible en: https://sisbib.unmsm.edu.pe/bvrevistas/parasitologia/v14_n1-2/pdf/a02v14n1-2.pdf

8. Gilbert Huaynate JT. Prevalencia y evaluación de la carga parasitaria de cerdos criados en los distritos de el Mantaro y San Lorenzo, provincia de Jauja,departamento de Junín [Internet]. Universidad Nacional Mayor de San Marcos; 2015. Disponible en: https://hdl.handle.net/20.500.12672/4610

9. Condemayta Z, Condemayta D, Ruelas D, Ibañez V. Prevalencia de Balantidium coli en la población humana y porcina asociado a factores socioeconómicos y saneamiento ambiental en el Distrito de Acora Puno Perú. Rev Investig Altoandinas. 22 de enero de 2018;20(1):85-94. doi:10.18271/ria.2018.332 
10. Mendoza-Gómez MF, Pulido-Villamarín A, Barbosa-Buitrago A, Aranda-Silva M. Presence of gastrointestinal parasites in swine and human of four swine production farms in Cundinamarca- Colombia. Rev MVZ Córdoba. 13 de noviembre de 2015;20(1):501427. doi:10.21897/rmvz.15

11. Cazorla Perfetti DJ, Acosta Quintero ME, Tortolero Low JL, Morales Moreno P. Prevalencia de enteroparásitos porcinos en una comunidad rural de la península de Paraguaná, estado falcón, Venezuela. Rev Científica la Fac Ciencias Vet [Internet]. 2013;23(1):19-25. Disponible en: https://produccioncientificaluz.org/index.php/cientifica/article/view/15770

12. Roepstorff A, Jorsal SE. Prevalence of helminth infections in swine in Denmark. Vet Parasitol. octubre de 1989;33(4):231-9. doi:10.1016/0304-4017(89)90133-7

13. Hassan Ahmed Hassan Ahmed I, Hyung-Kyu J, Yong-Man Y, Changhee D, Young-Ha L. Intestinal Parasite Infections in Pigs and Beef Cattle in Rural Areas of Chungcheongnam-do, Korea. Korean J Parasitol. 2010;48(4):347-9. doi:10.3347/kjp.2010.48.4.347

14. Tiwari KP, Chikweto A, Belot G, Vanpee G, Deallie C, Stratton G, et al. Prevalence of intestinal parasites in pigs in Grenada, West Indies. West Indian Vet J [Internet]. 2009;9(1):22-7. Disponible en: https://sta.uwi.edu/fms/vet/documents/4.pdf

15. Nissen S, Poulsen IH, Nejsum P, Olsen A, Roepstorff A, Rubaire-Akiiki C, et al. Prevalence of gastrointestinal nematodes in growing pigs in Kabale District in Uganda. Trop Anim Health Prod. 19 de marzo de 2011;43(3):567-72. doi:10.1007/s11250-0109732-х

16. Urquhart G, Armour J, Duncan J, Dun A, Jennings F. Parasitología veterinaria. ACRIBIA, editor. 2001.

17. Thrusfield M. Epidemiología veterinaria. ACRIBIA, editor. 1990.

18. Díaz Pasapera GM. Prevalencia de ascaris Suum en ganado porcino criollo (sus scrofa domestica) en la localidad Lagunas Mocupe, Provincia Chiclayo, Lambayeque 2017 [Internet]. Universidad Nacional Pedro Ruiz Gallo; 2018. Disponible en: https://hdl.handle.net/20.500.12893/2352

19. Arellano Cedillo AYM. Índices parasitológicos de las poblaciones y comunidades parasitarias gastrointestinales de cerdos (sus scrofa domesticus), durante el periodo agosto - noviembre 2014 en el distrito de Laredo - La Libertad [Internet]. Universidad Nacional de Trujillo; 2015. Disponible en: http://dspace.unitru.edu.pe/handle/UNITRU/8809

20. Sánchez Cárdenas H. Factores de riesgo y perfil epidemiológico de la cisticercosis porcina/teniasis humana en el distrito de Zapatero - 2019 [Internet]. Universidad Nacional de San Martín; 2019. Disponible en: http://hdl.handle.net/11458/3563 
21. Arizono N, Yoshimura Y, Tohzaka N, Yamada M, Tegoshi T, Onishi K, et al. Ascariasis in Japan: is pig-derived Ascaris infecting humans? Jpn J Infect Dis [Internet]. 2010;63(6):447-8. Disponible en: https://www.niid.go.jp/niid/images/JJID/63/447.pdf

22. Betson M, Nejsum P, Bendall RP, Deb RM, Stothard JR. Molecular epidemiology of ascariasis: a global perspective on the transmission dynamics of ascaris in people and pigs. J Infect Dis. 15 de septiembre de 2014;210(6):932-41. doi:10.1093/infdis/jiu193

23. Monteiro KJL, Calegar DA, Santos JP, Bacelar PAA, Coronato-Nunes B, Reis ERC, et al. Genetic diversity of Ascaris spp. infecting humans and pigs in distinct Brazilian regions, as revealed by mitochondrial DNA. Chiang T-Y, editor. PLoS One. 24 de junio de 2019;14(6):1-13. doi:10.1371/journal.pone.0218867

24. Zhou C, Chen J, Niu H, Ouyang S, Wu X. Study on the population evolution of Ascaris lumbricoides and Ascaris suum based on whole genome resequencing. Vet Parasitol. marzo de 2020;279:109062. doi:10.1016/j.vetpar.2020.109062

25. Melara Lagos K del R, Gutiérrez López NE. Prevalencia de parásitos gastrointestinales en porcinos de desarrollo en dos unidades de producción de la Universidad Nacional Agraria, finca Sta. Rosa, 2016 [Internet]. Universidad Nacional Agraria; 2017. Disponible en: http://repositorio.una.edu.ni/id/eprint/3646

26. Luna LA, Kyvsgaard N. Ocho diferentes especies de parásitos gastrointestinales fueron identificadas en cerdos de traspatio en El Municipio de El Sauce - León. Nicaragua. Rev Electrónica Vet [Internet]. 2005;6(10):1-9. Disponible en: https://www.redalyc.org/pdf/636/63617978020.pdf

27. Sánchez Salvador G. Identificación de parásitos gastrointestinales, hepáticos y renales en cerdos faenados en el matadero municipal de Huánuco en el período de mayo y junio 2015 [Internet]. Universidad Nacional Hermilio Valdizán; 2016. Disponible en: http://repositorio.unheval.edu.pe/handle/UNHEVAL/1333

\section{Conflicto de intereses}

Los autores declaramos que no existen conflictos de interés.

\section{Contribuciones de los autores}

Víctor Puicón: Procesamiento de muestras y análisis estadístico de los datos obtenidos, redacción del artículo.

Alicia López-Flores: Análisis estadístico, contribución con el procesamiento de muestras.

Fredy Fabian-Dominguez: Contribución en el diseño y redacción del artículo. 
Vol. 1, Núm. 1, jul-dic, 2021

Hugo Sánchez-Cárdenas: Muestreo y recolección de muestras coprológicas, coordinación con las autoridades y criadores de porcinos. 